\title{
Joint amyloid presenting as 'polymyalgic' rheumatoid arthritis
}

\author{
H. A. BIRD \\ From the Royal National Hospital for Rheumatic Diseases, Bath
}

SUMMARY. A patient presenting with 'polymyalgic' rheumatoid arthritis was shown to have synovial amyloid at an early stage of his illness. It is suggested that such patients merit early synovial biopsy and investigation.

Rheumatoid arthritis in the elderly occasionally presents with girdle stiffness mimicking polymyalgia rheumatica (Kersley, 1956; Huskisson and Hart, 1973). This must be distinguished from polymyalgia with synovitis where the Rose test is usually negative and radiological erosions are absent (Henderson et al., 1975). A patient is described with 'polymyalgic' rheumatoid where joint amyloid was identified on synovial biopsy taken at arthroscopy at an early stage of the illness.

\section{Case report}

A 71 year old retired miner presented with a 6 month history of bilateral shoulder pain and stiffness. His haemoglobin was $13 \cdot 2 \mathrm{~g} / \mathrm{dl}$, plasma viscosity was 1.86 centipoises (cP), and latex fixation negative. A provisional diagnosis of polymyalgia rheumatica was made though his symptoms improved with aspirin and physiotherapy.

Over the following 6 months his wrists, fingers, and shoulders became stiff and his knees swollen. Examination showed tenderness of the shoulder muscles and active synovitis at the wrists and knees. Rose-Waaler sheep cell test was now strongly positive (titre $1 / 512$ ) and $x$-rays of the hands showed carpal cysts (Fig. 1). These were thought not to be rheumatoid erosions even though they corresponded with one site of his synovitis. Synovial fluid aspirated from the knee had a white cell count of $3.0 \times 10^{\circ}$ (mainly mononuclear cells) and a positive RoseWaaler test (titre 1/32). A diagnostic arthroscopy 10 months after the onset of the symptoms showed

Accepted for publication November 20, 1977

Correspondence to Dr Bird, Rheumatism Research Unit, School of Medicine, 36 Clarendon Rd., Leeds LS2 9PJ. a mild non-specific synovitis without distinguishing features. Synovial histology showed areas of mild inflammation without crystal deposition. Haemotoxylin and eosin staining showed areas of eosinophilic degenerative change which showed pale green birefringence. Congo red and Sirius red staining also showed apple green birefringence confirming the presence of synovial amyloidosis (Fig. 2).

The patient was recalled for further investigations. $\mathrm{He}$ had no hepatosplenomegaly nor evidence of amyloid at other sites. There was no clinical evidence of malignancy or chronic infection. He had no proteinuria and a normal renal function. A rectal biopsy showed no evidence of amyloid. There was no radiological evidence of myeloma and light chains were not found in concentrated urine. Serum electrophoresis and immunoglobulins were normal though bone marrow aspiration was not performed, and cryoprecipitate not looked for. His serum Rose-Waaler remained strongly positive.

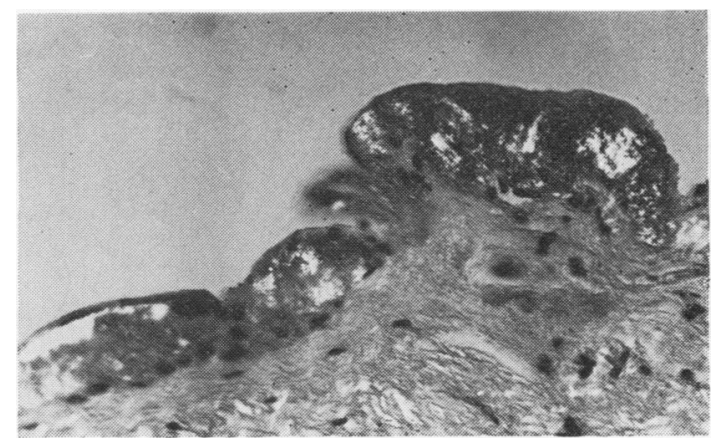

Fig. 1 Synovial biopsy showing amyloid. Sirius red staining with polarised light $(\times 80)$ 

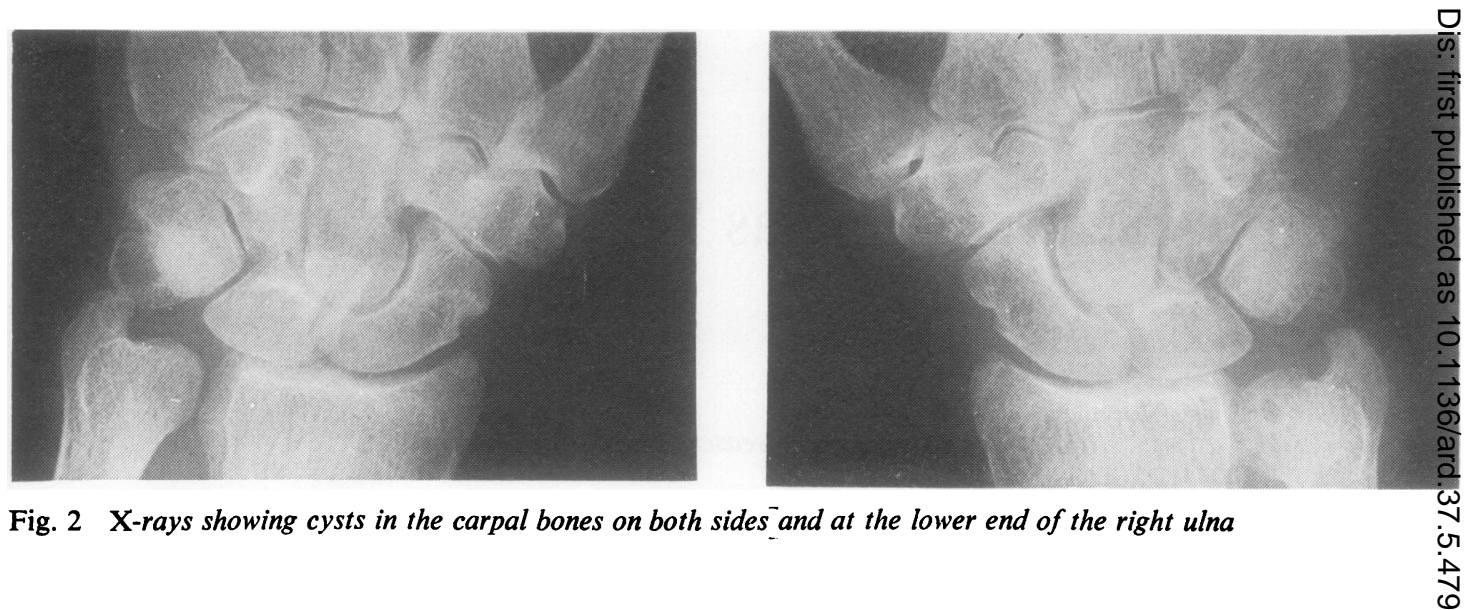

The patient's arthritis deteriorated and his symptoms could only be controlled by prednisolone (15 mg a day). Two years after the onset of symptoms he died suddenly at home from bronchopneumonia. Histological specimens were not saved from the necropsy.

\section{Discussion}

Joint amyloidosis can mimic rheumatoid arthritis and the majority of reported cases have progressed to myeloma (Cohen and Canoso, 1975). We found no obvious evidence of myeloma though the investigations did not exclude it, death from infection and a positive Rose-Waaler test both being compatible with myeloma. There was no clinical evidence to suggest the amyloid was secondary to chronic infection.

The alternative explanation is of primary joint amyloid. Although secondary amyloid may result from rheumatoid disease, we have not seen this

degree of amyloid on synovial biopsy before even in long-standing rheumatoid patients who presented in the conventional manner. It is suggested th@ patients with an atypical or polymyalgic presentatiof of rheumatoid arthritis deserve full investigatiof with synovial histology.

Dr N. G. Sanerkin, Bristol Royal Infirmary pe̊formed histological studies and Dr A. St. J. Dixfl gave permission to report the case."

\section{References}

Cohen, A. S., and Canoso, J. J. (1975). Rheumatologica aspects of amyloid, Clinics in Rheumatic Diseases, $\Phi$ 149-161.

Henderson, D. R. F., Tribe, C. R., and Dixon, A. St. 受 (1975). Synovitis in polymalgia rheumatica. Rheumatology and Rehabilitation, 14, 244-250.

Huskisson, E. C., and Hart, F. D. (1973). Joint Diseaš p. 81. John Wright \& Son, Bristol.

Kersley, G. D. (1956). Anarthritic rheumatoid disease (Letter.) Lancet, 2, 840. 